\title{
Clasificación molecular del carcinoma de colon y recto. Una revisión corta
}

\author{
Leonardo Saúl Lino-Silva, Alberto León-Takahashi, Horacio López-Basave, Alejandro Padilla-Rosciano, \\ Gerardo Miranda-Dévora, Martín Granados-García y Rosa Salcedo-Hernández \\ Secretaría de Salud, Instituto Nacional de Cancerología, Ciudad de México, México
}

\begin{abstract}
Resumen
Tradicionalmente las clasificaciones de los carcinomas se han basado en características clínicas o patológicas. Sin embargo, en las últimas décadas, con el desarrollo de la biología molecular, cada vez más tumores se están estudiando genéticamente y en varios se han creado clasificaciones moleculares (la más estudiada y utilizada es la de cáncer de mama). El cáncer de colon y recto no es la excepción. En esta revisión corta se explica la evolución de la clasificación molecular del cáncer de colon y recto y se abordan los conclusiones consensuadas al respecto.
\end{abstract}

PALABRAS CLAVE: Cáncer de colon y recto. Clasificación molecular. Inestabilidad microsatelital.

\begin{abstract}
Traditionally, carcinoma classifications have been based on clinical or pathological features. However, with the development of molecular biology in recent decades, more tumors are increasingly being genetically studied and, in several of them, molecular classifications have been created (the most widely studied and used is that for breast cancer). Colon and rectum cancer are no exception. In this short review, the evolution of colon and rectum cancer molecular classification is explained and the consensus conclusions on the subject are addressed.
\end{abstract}

KEY WORDS: Colon and rectum cancer. Molecular classification. Microsatellite instability.

\section{Introducción}

Tradicionalmente, los cánceres se han clasificado en función del estadio clínico y de sus características histológicas. En el cáncer de colon y recto (CCR) se ha demostrado que el pronóstico depende de la afectación tumoral local, la metástasis ganglionar regional, la invasión linfovascular, los márgenes quirúrgicos positivos, la elevación preoperatoria del antígeno carcinoembrionario y el grado tumoral. Sin embargo, la respuesta al tratamiento todavía es difícil de predecir en escenarios específicos, en particular en la etapa metastásica. ${ }^{1}$ Los sistemas de estadificación Tumor, Node, Metastasis (TNM) de la Unión Internacional de Control del Cáncer y la del American Joint Committee on Cancer son los más utilizados para el cáncer colorrectal. ${ }^{2,3}$ La clasificación TNM es un sistema dual y comprende una clasificación clínica o pretratamiento y una patológica o posquirúrgica. ${ }^{4}$ Las decisiones quirúrgicas se basan en la clasificación clínica, mientras que el manejo posquirúrgico y el pronóstico se establecen utilizando la clasificación patológica.

En el CCR no se ha implementado todavía una clasificación molecular estandarizada internacionalmente, sin embargo, existen diferentes aspectos patogénicos
Fecha de recepción: 13-04-2017

Fecha de aceptación: 15-01-2018

DOI: 10.24875/GMM.18003411
Gac Med Mex. 2018;154:598-604

Disponible en PubMed www.gacetamedicademexico.com 
que podrían servir de base. ${ }^{5}$ Con el advenimiento de la alta tecnología molecular se están generando numerosos datos genómicos, transcriptómicos, proteómicos y metabolómicos que están facilitando la caracterización molecular integral del cáncer. El objetivo es identificar una firma molecular que se pueda aplicar en la clínica para el tratamiento y cuidado personalizado del paciente con cáncer. Además, sería útil establecer firmas moleculares capaces de identificar a los individuos con mayor riesgo de desarrollar la enfermedad (biomarcadores de diagnóstico), para poder tomar medidas preventivas. Las firmas moleculares también podrían guiar a los médicos respecto a la predicción de la recurrencia de la enfermedad después de la cirugía (biomarcadores de vigilancia).

Por su parte, los biomarcadores pronósticos dan una indicación de la probable progresión de la enfermedad y los biomarcadores predictivos orientan hacia las probables respuestas al tratamiento. Lo anterior ha sido demostrado en el cáncer de mama, ${ }^{6-}$ ${ }^{9}$ cuyo diagnóstico y tratamiento en algunos casos se basan en la expresión de ciertas moléculas, como receptores de estrógenos para indicar el tratamiento antiestrógeno o la amplificación del receptor 2 del factor de crecimiento epidérmico humano para el tratamiento dirigido a este. ${ }^{10}$ Este progreso en el cáncer de mama ha sido impulsado por su clasificación molecular, lo cual no ha sucedido en el CCR, en el que la caracterización molecular se ha quedado atrás.

Aunque las clasificaciones moleculares publicadas respecto al CCR parecen ser diferentes, hay factores en común. En esta revisión, se resalta la evolución de las diferentes clasificaciones propuestas.

\section{Clasificaciones moleculares iniciales}

Tres principales vías están implicadas en la clasificación molecular del CCR: inestabilidad de los microsatélites (IMS), inestabilidad cromosómica (IC) y metilación de las islas CpG (MICpG). La IMS se caracteriza por pequeñas inserciones 0 deleciones en secuencias repetitivas del ADN (microsatélites); 11 cuando la IMS no está presente, un tumor se considera como estable en microsatélites (MSS). La IC se define como la frecuencia elevada de IMS y segregación de cromosomas enteros; la MICpG se produce cuando hay metilación de las islas CpG en la región promotora y se asocia con el silenciamiento transcripcional..$^{12,13}$
En 2007, Jass ${ }^{11}$ propuso una clasificación molecular del cáncer colorrectal incluyendo KRAS, BRAF, reparación por apareamiento incorrecto, MICpG y estado mutacional del gen MGMT. Desde entonces, se han publicado numerosos estudios pronósticos y predictivos sobre este tema, centrados en un solo marcador molecular. En 2008, Ogino y Goel propusieron una clasificación basada en los seis subgrupos propuestos por Jass:11-14

- Grupo 1, IMS-H MICpG-alta (10\%).

- Grupo 2, IMS-H MICpG-baja (5\%).

- Grupo 3, IMS-L/MSS MICpG-alta (5-10\%).

- Grupo 4, IMS-L MICpG-baja (5\%).

- Grupo 5: MSS MICpG-baja (30 a $35 \%$ ).

- Grupo 6: IMS-L/MSS MICpG-0 ( $40 \%$ ).

Esta clasificación molecular depende de los marcadores del ADN común y del estado mutante de BRAF y KRAS. ${ }^{15}$

\section{Clasificación mesenquimal/epitelial}

En 2012, Schlicker et al. usaron un panel de 74 líneas diferentes de células de CCR. ${ }^{16}$ Utilizando el agrupamiento iterativo, este análisis reveló cinco subtipos: 1.1, 1.2, 1.3, 2.1 y 2.2, que se validaron con éxito en más de 1600 muestras de tumores de CCR de conjuntos de datos de expresión génica disponibles públicamente. Los tumores tipo 1 mostraron una firma de expresión mesenquimatosa, tenían un mal pronóstico y un número similar de tumores con IMS y MSS. Los tumores tipo 2 presentaban expresión epitelial, MSS y tenían un buen pronóstico.

La subtipificación adicional de los tumores tipo 1 reveló que el subtipo 1.1 era fuertemente mesenquimatoso, se presentaba en fase tardía y los genes regulados estaban implicados principalmente en la señalización del calcio; además, el subtipo 1.1 se caracterizó por activación de vías implicadas en la angiogénesis, la inflamación y la proliferación. Más mujeres presentaron el subtipo 1.2; en su mayoría, los tumores eran IMS y tenían vías similares a 1.1, pero también activaban fuertemente la vía de señalización JAK-STAT. El subtipo 1.3 mostró alta expresión de genes transportadores y fueron principalmente MSS.

La estratificación del tipo 2 dio lugar a los subtipos 2.1 y 2.2. En el primero se activaron vías relacionadas con la inflamación, la angiogénesis y la proliferación. En el subtipo 2.2, los genes regulados eran los implicados en el ciclo celular y la síntesis de aminoácidos. 
Los CCR clasificados como subtipo 1.2 fueron muy sensibles a la glucógeno sintasa quinasa, Src y los inhibidores de la vía de señalización de Wnt. Por otro lado, las líneas celulares de CCR designadas como tipo 2 eran más sensibles a los inhibidores de la cinasa aurora.

\section{Clasificación basada en IC, IMS y el estado mutacional de KRAS/BRAF}

En 2013, dos artículos publicados en Nature Medicine revolucionaron la clasificación molecular. De Sousa et al. describieron tres subtipos de CCR molecularmente distintos: CCS1, CCS2 y CCS3, en los que se caracterizó por primera vez CCS3. ${ }^{17}$ Una parte integral de este estudio fue el análisis de los datos de expresión génica de 1164 pacientes con CCR. Todas las muestras de CCR, xenoinjertos, líneas celulares y lesiones precursoras se distribuyeron en los tres subtipos usando un clasificador de 146 genes. CCS1 principalmente se compone de cánceres cromosómicos inestables (IC) que se encuentran principalmente en el lado izquierdo del colon. Los genes diana Wnt estuvieron altamente expresados y los tumores metastásicos fueron menos frecuentes en comparación con los tumores CCS3. Los tumores CCS2 fueron positivos a IMS/MICpG y estuvieron ubicados en el lado derecho del colon. Hay relativamente poca información sobre CSS2.

Por su parte, en CCS3 no clasificaron tumores con IC o IMS, pero había un número relativamente grande de pacientes con mutaciones en KRAS o BRAF. Los tumores se distribuyeron a lo largo del colon y tendieron a ser poco diferenciados. Con base en el estado de MSS y MICpG+, junto con la relativa sobrerrepresentación de mutaciones en $B R A F$, se formuló la hipótesis de que estos tumores pueden surgir de la ruta dentada. Por otra parte, al comparar CCS1 con CCS3 utilizando Gene Set Enrichment Analysis, parecía que en el subtipo CCS3 los genes regulados estaban involucrados en la transición epitelial a mesenquimal, remodelación de la matriz, migración celular y señalización del factor de crecimiento transformante $\beta$. Los autores concluyeron que los tumores CCS3 eran altamente malignos en comparación con CCS1 y CCS2.

Además de la clasificación propuesta, los autores examinaron las respuestas de los diferentes subtipos al tratamiento dirigido. Los pacientes con tumor CCS3 metastásico eran resistentes al cetuximab, independientemente del estado mutacional de KRAS. Esta diferencia también se observó in vitro. ${ }^{17}$
Sadanandam et al. describieron seis subtipos junto con su respuesta al cetuximab, la quimioterapia y la supervivencia libre de enfermedad (DFS). Los subtipos se generaron mediante un consenso basado en la agrupación de los perfiles de expresión génica de 1290 muestras: $^{18}$

- Subtipo tallo: el menos diferenciado, se asoció con la base de la cripta del colon y con mala DFS. Los pacientes tuvieron mayor expresión de la vía de señalización de Wnt, células madre, genes mioepiteliales y mesenquimales y menor expresión de los marcadores de diferenciación. Se predijo que este grupo se beneficiaría de la quimioterapia (en especial de un régimen a base de leucovorina cálcica, 5-fluorouracilo e irinotecan-FOLFIRI).

- Subtipo inflamatorio: no se asoció con cript-top o fenotipos de base. Los principales genes regulados en este grupo estuvieron relacionados con interferón y quimiocinas. Conforme a lo anterior, como tratamiento adyuvante los pacientes deben idealmente recibir quimioterapia, más específicamente con FOLFIRI. Por otro lado, en los tumores metastásicos podrían ser ineficaces la quimioterapia y el cetuximab. En este subtipo se identificó DFS intermedia.

- Subtipo goblet: se encontró en la parte superior de la cripta de colon y se relacionó con buena DFS. Este subtipo exhibió alta expresión de ARNm de MUC2 y TFF3 específicas de calabaza. Es posible que los pacientes no respondan al tratamiento adyuvante y, por lo tanto, se sugirió implementar una vigilancia estrecha después de la resección quirúrgica. En la etapa metastásica se debe administrar quimioterapia (preferiblemente FOLFIRI).

- Subtipo enterocito: se caracterizó por alta expresión de genes específicos de enterocitos. Los pacientes asignados a este grupo tuvieron una DFS intermedia. Con base en su firma génica, se determinó que estos tumores se identifican con el fenotipo de criptas y crestas en el colon. Comparado con el fenotipo de células madre o progenitoras, es más diferenciado. Los autores recomendaron que los pacientes que recibían tratamiento adyuvante debían ser tratados con quimioterapia (preferiblemente FOLFIRI) o con otra opción (excepto cetuximab o inhibidores de c-MET). En cuanto a la enfermedad metastásica, los autores no recomendaron la quimioterapia, el cetuximab ni inhibidores de c-MET. 
- Subtipo de amplificación de tránsito (AT): se consideró heterogéneo ya que tanto las células madre como los genes diana de Wnt se expresaron de forma irregular. Este subtipo también exhibió un fenotipo mixto: $59 \%$ de los tumores tenía una firma de cripta alta y baja expresión de la vía de señalización de Wnt. Se asoció significativamente con la base de cripta, tallo expresado y marcadores progenitores como LGR5 y ASCL2. Los autores recomendaron evitar la quimioterapia adyuvante y la quimiorradioterapia, ya que identificaron que los pacientes con este subtipo tenían menor DFS.

Después de una serie de ensayos de proliferación para monitorizar las respuestas de fármacos, el subtipo AT se dividió:

- Subtipo de amplificación-tránsito sensible a cetuximab (AT-SC).

- Subtipo de tránsito-amplificación resistente a cetuximab (AT-CR).

AT-SC mostró mayor expresión de EREG y AREG en comparación con AT-CR, en el cual se observó mayor expresión de la filamina $A$, involucrada en la regulación de c-MET. Los autores concluyeron que ante metástasis, los pacientes con AT-SC deben ser tratados con cetuximab mientras que con AT-RC debe ser tratado con un inhibidor de c-MET.

\section{Clasificación basada en variables clínico-patológicas}

En 2013, Marisa et al. publicó una clasificación de la expresión génica de seis subtipos moleculares basados en las variables clínico-patológicas comúnmente utilizadas como marcadores de ADN. ${ }^{15}$ La relevancia de esta clasificación es que los subtipos se asociaron con diferentes pronósticos. El estudio se realizó con 443 pacientes y un grupo de validación de 1029 pacientes. Los subtipos se nombraron de acuerdo con sus características biológicas.

El subtipo $\mathrm{C} 1$, denominado IC-immune-down, compartió numerosas similitudes con C5 IC-Wnt-up, ambos en la vía convencional del precursor de la neoplasia, con frecuencia muy baja de genes deficientes en la reparación de la falta de concordancia (dMMR) y de MICpG, en contraste con la alta frecuencia de IC. La mayoría se localizó en el colon distal. Respecto al estado mutacional, C1-IC-immune-down y C5 ICWnt-up exhibieron frecuencia intermedia de mutación de KRAS y TP53 y mutación muy baja de BRAF. Una de las principales diferencias fue que C5-IC-Wnt-up se asoció con una frecuencia intermedia de fenotipo de células madre similar a los perfiles de expresión génica, mientras que C1-IC-immune-down exhibió muy baja frecuencia.

Los otros cuatro subtipos, C2 dMMR, C3 KRASm, C4 CSC y C6 IC-norm-L, se vincularon a la ruta de la neoplasia precursora dentada. El subtipo C2 dMMR implicó principalmente mutaciones dMMR (68\%) y en BRAF (40\%); se localizaron muy frecuentemente en el colon proximal y se registró una frecuencia muy alta de MICpG (59\%). Se encontraron tumores mutantes KRAS con una frecuencia intermedia. Respecto a la expresión de genes cuya selección fue supervisada, la mayoría de los C2 dMMR exhibió una firma tipo BRAF y una firma dentada. Además, se encontró que el sistema inmunológico y las vías de crecimiento celular estaban reguladas positivamente en este subtipo, mientras que la vía de señalización Wnt estaba regulada negativamente.

El subtipo C3KRASm también se localizó frecuentemente en el colon proximal e incluyó principalmente tumores KRAS mutantes (87\%). La mayoría de las vías de señalización en los tumores C3 KRASm fueron reguladas por inhibición.

El subtipo C4-CSC con metástasis (31\%) fue el único con una asociación reproducible entre el mal pronóstico y la expresión de genes de células madre. Este subtipo se localizó frecuentemente en el colon proximal, tuvo una frecuencia intermedia de $\mathrm{IC}+\mathrm{y}$ $34 \%$ de los pacientes presentaron MICpG+. Se informaron frecuencias intermedias de mutaciones en KRAS y TP53. La mayoría exhibió fenotipo de célula madre como firma de expresión génica $(91 \%)$.

El subtipo final descrito en este estudio fue C6 ICnorm-L. Aunque se clasificó bajo la categoría de neoplasia precursora dentada, fue principalmente IC+ (86\%), MICpG-, TP53 mutante y se localizó en el colon distal. En contraste con los otros subtipos IC+, exhibió frecuencia muy alta de la firma de expresión génica normal e intermedia del perfil de expresión génica cerrada.

\section{Clasificación integral}

En julio de 2013, Budinska et al. describió una clasificación de cinco subgrupos utilizando 1113 perfiles de expresión génica de CCR y 720 perfiles transcriptómicos de CCR..$^{19}$ Los autores usaron el análisis del transcriptoma del genoma en su conjunto y describieron las características biológicas, morfología, 
variables clínicas comunes y marcadores moleculares de cada subtipo:

- Subtipo de tipo cripta superficial (A): se enriqueció significativamente en KRAS, con histopatología papilar o dentada predominante y bajo porcentaje de núcleos positivos a $\beta$-catenina en el frente invasivo. Los autores también observaron que estaba bien diferenciado y que era comparable al epitelio de colon normal por perfiles de expresión génica. En este subtipo, la supervivencia después de la recaída fue de 28.9 meses.

- Subtipo de tipo cripta inferior (B): bien diferenciado, mostró morfología tubular compleja y tuvo alto porcentaje de núcleos positivos a $\beta$-catenina en el frente invasivo. Se registró mayor ganancia/ amplificación del número de copias en el cormosoma 20q. Por otra parte, se detectó sobreexpresión de los genes implicados en la parte superior de la cripta del colon, proliferación (principalmente EREG) y vía de señalización Wnt. Por el contrario, la expresión génica que regulaba la transición epitelial mesenquimal/estroma, el sistema inmunitario y las células secretoras fueron reguladas por inhibición. Los tumores se localizaron principalmente en el lado izquierdo y fueron grado 2. La supervivencia después de la recaída fue de 50.4 meses.

- Subtipo C, tipo MICpG-H: comúnmente mutante IMS+ y BRAF (87\%), caracterizado por patrones de crecimiento sólido/trabecular o mucinoso. Comúnmente, los tumores se encuentran en el lado derecho y son de alto grado. En el frente invasivo no mostraron inmunorreactividad nuclear de प-catenina, aunque transcriptómicamente tenían alta expresión de genes asociados con la inmunidad, metalotioneínas y módulo de genes homeobox. Se asoció con supervivencia pobre (de 6.9 meses después de la recaída). Se especula que una vez que hacen metástasis es resistente a la quimioterapia.

- Subtipo CCR-mesenquinales: el examen histopatológico reveló un patrón desmoplásico y la inmunoquímica, que solo un bajo porcentaje de núcleos positivos a $\beta$-catenina estaban presentes en el frente invasivo. Este subtipo también tenía alta expresión de genes transición epitelial mesenquimal/estroma. Por el contrario, la expresión de los genes de síntesis de lípidos y la vía de señalización Wnt de las firmas objetivo fueron entre los down-regulados en el subtipo mesenquimal. Además, tenía alta frecuencia de mutaciones en BRAF y se correlacionó con mal OS, posiblemente como resultado de la alta expresión de la transición epitelial mesenquimal y baja expresión de la proliferación de genes asociados. Además, fue pronóstico respecto a la supervivencia libre de recaída.

- Subtipo mixto (E): se localizó principalmente en el lado izquierdo del colon. Al examen histopatológico se observó complejo tubular y la inmunohistoquímica mostró un alto porcentaje de núcleos positivos a $\beta$-catenina en el frente invasivo. Este subtipo también tenía expresión génica de alta transición epitelial mesenquimal/estroma, vía de señalización Wnt firmas de destino, EREG gen módulo de expresión, y homeobox gen módulo de expresión.

\section{Clasificación basada en características biológicas del cáncer}

En 2013, Roepman et al. ${ }^{20}$ propusieron tres subtipos intrínsecos diferentes basados en las características biológicas de la transición de cáncer epitelial a mesenquimal, deficiencia en los genes de reparación de desajuste y proliferación celular. Esta clasificación se derivó de datos multiómicos generados a partir de 188 pacientes con CCR en fases I a IV. El conjunto de validación estuvo integrado por 320 pacientes en estadio II y 223 en estadio III y se asoció con el pronóstico y la respuesta a la quimioterapia; 35 y $22 \%$ de los pacientes clasificaron como tipo $\mathrm{A}$ epitelial con deficiencia de MMR en la cohorte inicial y en la cohorte de validación, respectivamente. Se observó un porcentaje significativamente mayor de tumores IMS+ (49\%) y de mutaciones de BRAF; $68 \%$ de los pacientes presentó un perfil de expresión IMS/dMMR; también se observó expresión más alta de Ki67 cuando se comparó con los otros dos subtipos. El pronóstico para los pacientes con IMS tipo A fue muy bueno (93\% con 10 años de supervivencia sin metástasis). La respuesta a la quimioterapia se evaluó en 222 pacientes en estadio III que tuvieron ME mejor de la quimioterapia adyuvante, con una razón de riesgo (HR) de $0.39(p=0.18)$.

El $52 \%$ de la cohorte experimental y $62 \%$ de la de validación clasificaron con epitelio proliferativo subtipo B. Este subtipo abarcó principalmente tumores con $B R A F$ de tipo salvaje (98\%), casi enteramente fenotipo MSS y pMMR (99\%). Los marcadores mesenquimales CDH2, FGFR1, y TGFB1 fueron inhibidos, mientras que cuatro marcadores epiteliales fueron activados. Este subtipo se caracterizó como 
altamente proliferativo. Los pacientes tenían un pronóstico basal relativamente pobre, pero se esperaba que se beneficiaran con la quimioterapia adyuvante a base de 5-fluorouracilo ( $H R=0,42, p=0,014$ ).

Correspondieron al subtipo $C$ mesenquimal, $13 \%$ de la cohorte experimental y $17 \%$ de la cohorte de validación. Una pequeña proporción mostró $B R A F$ mutante (16\%) y $36 \%$ dMMR. Todos los marcadores mesenquimales, con excepción de FLT1, fueron considerablemente regulados en comparación con los marcadores epiteliales CDH, EGFR y MET, regulados a la baja. Además, se registró baja actividad proliferativa en este grupo. Los pacientes tenían mal pronóstico y eran resistentes a la quimioterapia con 5 -fluorouracilo.

\section{Clasificación molecular consensuada}

En 2015 se publicó The Consensus Molecular Subtypes of Colorectal Cancer, ${ }^{21}$ en el que se identificaron cuatro subtipos moleculares. Los 18 conjuntos de datos utilizados en los estudios de caracterización y subtipificación incluyó a 4151 pacientes. Los autores dividieron la cohorte en dos grupos equivalentes para su validación.

Los cuatro grupos de subtipificación de consenso incluyeron 3104 muestras; 858 (13\%) no correspondieron a ningún subtipo y, por lo tanto, se describieron como no clasificables.

- Consenso molecular subtipo 1 (CMS1): $14 \%$ de la cohorte correspondió a este subtipo, el cual muestra inestabilidad de microsatélites, IMS+, MICpG-H, fenotipo hipermutado y con numeraciones mutaciones en BRAF. El subtipo inmune de IMS se caracterizó por una expresión aumentada de genes asociados con infiltrados inmunes y fuerte activación de las vías de evasión inmune. Estos tumores fueron frecuentes en las mujeres, en el lado derecho, de presentación en estadios posteriores y con peor supervivencia después de la recaída.

- Consenso molecular subtipo 2 (CMS2): $37 \%$ de la cohorte clasificó en este subtipo canónico-epitelial. CMS2 tuvo las mayores ganancias y pérdidas de número de copias; además, tenía alta expresión de Wnt y MYC downstream objetivos. Estos tumores se presentan comúnmente en el lado izquierdo. Los pacientes tuvieron mejores tasas de supervivencia después de la recaída en comparación con CMS1.

- Consenso molecular subtipo 3 (CMS3): $13 \%$ de la cohorte clasificó en este subtipo. La mayoría de los tumores presentaban IMS mixta, principalmente MICpG-L, y tenían baja frecuencia de alteraciones en el número de copias. Las mutaciones en KRAS fueron comunes.

- Consenso subtipo molecular 4 (CMS4): $23 \%$ de la cohorte clasificó en este subtipo mesenquimal, caracterizado por prominente actividad del factor de crecimiento transformante- $\beta$, invasión estromal y angiogénesis. Tuvo alta frecuencia de ganancias y pérdidas en el número de copias. Los tumores tienden a ser diagnosticados en una etapa posterior y muestran la mayor recaída y la menor supervivencia global.

\section{Conclusión}

La clasificación molecular del cáncer colorrectal es de gran importancia, ya que la identificación de subtipos sirve para generar y relacionar parámetros clínico-patológicos, supervivencia global, supervivencia libre de progresión y respuesta al tratamiento y recaída. Estas correlaciones pueden facilitar la comprensión de la biología del cáncer colorrectal, proporcionar pruebas respecto a la carcinogénesis y ayudar a descubrir nuevos subtipos moleculares. Una clasificación molecular es de suma importancia para el descubrimiento y la implementación clínica de biomarcadores pronósticos y predictivos y hacer realidad la atención sanitaria personalizada.

\section{Bibliografía}

1. Compton CC, Fielding LP, Burgart LJ, Conley B, Cooper HS, Hamilton SR, et al. Prognostic factors in colorectal cancer. College of American Pathologists Consensus Statement 1999. Arch Pathol Lab Med. 2000;124(7):979-994.

2. Edge SB, Byrd DR, Compton CC, Fritz AG, Greene FL, Trotti A, editores. AJCC Cancer Staging Manual. Octava edición. EE. UU.: Springer; 2010.

3. Edge SB, Compton CC. The American Joint Committee on Cancer: the $7^{\text {th }}$ edition of the AJCC Cancer Staging Manual and the future of TNM. Ann Surg Oncol. 2010;17(6):1471-1474.

4. Labianca R, Beretta GD, Kildani B, Milesi L, Merlin F, Mosconi S, et al. Colon cancer. Crit Rev Oncol Hematol. 2010;74(2):106-133.

5. Haq Al, Schneeweiss J, Kalsi V, Arya M. The Dukes staging system: a corner stone in the clinical management of colorectal cancer. Lancet Oncol. 2009;10(11):1128.

6. Albain KS, Barlow WE, Shak S, Hortobagyi GN, Livingston RB, Yeh IT, et al. Prognostic and predictive value of the 21-gene recurrence score assay in postmenopausal women with node-positive, oestrogen-receptor positive breast cancer on chemotherapy: a retrospective analysis of a randomized trial. Lancet Oncol. 2010;11(1):55-65.

7. Paik S, Tang G, Shak S, Kim C, Baker J, Kim W, et al. Gene expression and benefit of chemotherapy in women with node-negative, estrogen receptor-positive breast cancer. J Clin Oncol. 2006;24(23):3726-3734.

8. Liu Z, Zhang XS, Zhang S. Breast tumor subgroups reveal diverse clinical prognostic power. Sci Rep. 2014;4:4002.

9. Rouzier R, Perou CM, Symmans WF, Ibrahim N, Cristofanilli M, Anderson $\mathrm{K}$, et al. Breast cancer molecular subtypes respond differently to preoperative chemotherapy. Clin Cancer Res. 2005;11(16):5678-5685.

10. Forda SR, Bergström R, Chlebus M, Barker R, Andersen PH. Priorities for improving drug research, development and regulation. Nat Rev Drug Discov. 2013;12(4):247-248. 
11. Jass JR. Classification of colorectal cancer based on correlation of clinical, morphological and molecular features. Histopathology. 2007;50(1):113-130.

12. Rajagopalan $H$, Nowak MA, Vogelstein $B$, Lengauer $C$. The significance of unstable chromosomes in colorectal cancer. Nat Rev Cancer. 2003;(9):695-701.

13. Lao VV, Grady WM. Epigenetics and colorectal cancer. Nat Rev Gastroenterol Hepatol. 2011;8(12):686-700.

14. Ogino S, Goel A. Molecular classification and correlates in colorectal cancer. J Mol Diagn. 2008;10(1):13-27.

15. Marisa L, De-Reyniès A, Duval A, Selves J, Gaub MP, Vescovo L, et al. Gene expression classification of colon cancer into molecular subtypes: characterization, validation, and prognostic value. PLoS Med. 2013;10(5):1-13.

16. Schlicker A, Beran G, Chresta CM, McWalter G, Pritchard A, Weston S, et al. Subtypes of primary colorectal tumors correlate with response to targeted treatment in colorectal cell lines. BMC Med Genomics. 2012;5(1):1-15.
17. De-Sousa-Melo F, Wang X, Jansen M, Fessler E, Trinh A, De-Rooij LP, et al. Poor-prognosis colon cancer is defined by a molecularly distinct subtype and develops from serrated precursor lesions. Nat Med. 2013;19(5):614-618.

18. Sadanandam A, Lyssiotis CA, Homicsko K, Collisson EA, Gibb WJ, Wullschleger $\mathrm{S}$, et al. A colorectal cancer classification system that associates cellular phenotype and responses to therapy. Nat Med. 2013;19(5):619-625

19. Budinska E, Popovici V, Tejpar S, D’Ario G, Lapique N, Sikora KO, et al. Gene expression patterns unveil a new level of molecular heterogeneity in colorectal cancer. J Pathol. 2013;231(1):63-76.

20. Roepman P, Schlicker A, Tabernero J, Majewski I, Tian S, Moreno V, et al. Colorectal cancer intrinsic subtypes predict chemotherapy benefit, deficient mismatch repair and epithelial-to-mesenchymal transition. Int $J$ Cancer. 2014;134(3):552-562.

21. Guinney J, Dienstmann R, Wang X, De-Reynies A, Schlicker A Soneson C, et al. The Consensus Molecular Subtypes of Colorectal Cancer. Nat Med. 2015;21(11):1350-1356. 